\title{
De novo cerebellar medulloblastoma in a 26-year recurrence-free case of temporal glioblastoma multiforme
}

\author{
Case report
}

\author{
Javier Fandino, M.D., Adrian M. Siegel, M.D., R. Hubert Laeng, M.D., \\ AND M. GAZI YAŞARGIL, M.D.
}

\begin{abstract}
Departments of Neurosurgery and Pathology, Kantonsspital Aarau, Aarau; Departments of Neurosurgery and Neurology, University Hospital Zurich, Switzerland; and Department of Neurosurgery, University of Arkansas for Medical Sciences, Little Rock, Arkansas
\end{abstract}

\begin{abstract}
The authors describe a patient who survived 26 years after resection of a right temporal glioblastoma multiforme (GBM) without signs of tumor recurrence. Preoperative emergency angiography demonstrated a hypovascular mass localized in the right temporal lobe with right-to-left shift of the vascular structures. At surgery, the tumor had cystic and solid components localized in the lateral occipitotemporal gyrus, reaching the posterolateral wall of the inferior horn of the right lateral ventricle and extending to the trigone and posterior horn. The initial pathological diagnosis of a GBM was reviewed and confirmed throughout the follow-up period. Twenty-six years after surgery and subsequent radiosurgery, the patient underwent resection of a medulloblastoma localized in the right cerebellum as well as stereotactic biopsy sampling of tissue at the original GBM site. Neither radiological nor histological evidence of recurrence of the GBM could be documented. The intraoperative, histological, and radiological findings are described.
\end{abstract}

\section{KEY WORDS • glioblastoma multiforme • long-term survival • medulloblastoma • adult}

The prognosis for patients with GBM remains poor despite multimodality treatment involving surgery, radiotherapy, and chemotherapy. A mean survival of 1 year is generally cited. ${ }^{13,45}$ Long-term survival in patients with GBM is uncommon and has been reported in 2 to $5 \%$ of such cases 3 years after diagnosis. ${ }^{10,42}$ Although evaluation of prognostic factors in GBM has been controversial, there is evidence that age younger than 45 years, multimodal therapy, complete resection, a long disease-free interval after the initial diagnosis, and high KPS score favor longer survival. ${ }^{1-6}$ Proliferative indices of diffusely infiltrating astrocytomas correlate with histological grades. ${ }^{8,24,33,36}$ Moreover, the presence of hypertriploid DNA multinucleated giant cells is associated with prolonged survival. ${ }^{7,38,39}$ Although the authors of some studies have reported a favorable prognosis associated with GBM if molecular alterations such as an increased frequency of TP53 mutations and deletions of chromosomal $\operatorname{arm} 17 \mathrm{p}$ are present, ${ }^{32,41,51}$ and a poorer prognosis if ampli-

Abbreviations used in this paper: CSF = cerebrospinal fluid; $\mathrm{CT}=$ computerized tomography; EGFR = epidermal growth factor receptor; FDG $=\left[{ }^{18} \mathrm{~F}\right]$ fluorodeoxyglucose; $\mathrm{GBM}=$ glioblastoma multiforme; KPS = Karnofsy Performance Scale; MR = magnetic resonance; $\mathrm{PET}=$ positron emission tomography; $\mathrm{POG}=$ Pediatric Oncology Group; WHO = World Health Organization. fication with overexpression of EGFR is evidenced, ${ }^{12,21}$ other investigators have not confirmed these findings. $5,26,27,40$

We presented a case of long-term survival in a patient 26 years after undergoing resection and radiotherapy of a right temporal and GBM who later presented with a new cerebellar medulloblastoma and no evidence of tumor recurrence.

\section{CASE REPORT}

History and First Examination. This 35-year-old righthanded man was admitted to the emergency department (University Hospital Bern, Switzerland) in October 1975 with severe, unremitting headaches, nausea, and slight left hemiparesis. Fundoscopy revealed papilledema. A homonymous left upper quadrantanopsia was present. The patient had a history of intermittent headaches since youth and had experienced six episodes of unconsciousness during the preceding 10 years, which may have been absence seizures. Shortly after admission, his level of consciousness deteriorated and he developed anisocoria and worsening left hemiparesis. After emergency right carotid artery angiography was performed, the patient was referred for surgical treatment to the University Hospital Zurich. The angiography study demonstrated a hypovas- 
cularized mass in the right temporal lobe with right-to-left shift of the vascular structures. Computerized tomography scanning was not available. Because of further neurological deterioration with symptoms consistent with intracranial hypertension (persisting vomiting, progressive somnolence, and bradycardia), the patient underwent intubation and was brought to the operating room.

First Operation. On exposure of the brain, the surgeon (M.G.Y.) observed edematous gyri with no signs of cortical pathological tissue or vascularization. Exploration with a needle revealed a cyst, localized in the medullary layer of the temporal lobe, which was punctured and drained. A total of $40 \mathrm{ml}$ of xanthochromic fluid was drained, after which the temporal lobe collapsed. The tumor was reached via a $2-\mathrm{cm}$ cortical incision in the middle temporal gyrus. Microsurgical exploration demonstrated a tumor localized in the lateral occipitotemporal gyrus, deeply subcortical and beneath the posterolateral wall of the inferior horn of the right lateral ventricle and extended to the trigone and posterior horn. Despite proximity, the tumor did not invade the hippocampus. The tumor was firm and fibrous, well circumscribed, and with little vascularization. Necrotic tissue with thrombosed veins was identified and easily resected using suction. Intraoperative inspection suggested macroscopic grosstotal tumor resection. The resection cavity measured $4 \times$ $5 \times 5 \mathrm{~cm}$.

First Postoperative Course. The only persisting postoperative deficit was a homonymous left upper quadrantanopsia. The postoperative course was uneventful and the patient was discharged 7 days after surgery. He underwent postoperative fractionated whole-brain radiation therapy (telecobalt) of $5100 \mathrm{cGy}$ with a local boost in the right temporoparietal region of $1440 \mathrm{cGy}$. Chemotherapy was not undertaken. Follow-up evaluations were conducted in a neurosurgical clinic on a yearly basis from 1975 to 1991 . In the subsequent years, the patient sporadically visited his family physician. The first postoperative contrastenhanced CT scan, obtained in 1978, demonstrated the resection cavity but no residual or recurrent tumor. During the follow-up period between 1978 and 1990, the patient underwent a total of nine sessions in which CT scans were obtained (Fig. 1). The first contrast-enhanced MR imaging study was performed in 1991, and thereafter the patient rejected further neuroimaging studies and was lost to neurosurgical follow up. During this time the patient's KPS score improved to 90 . He resumed his job as a computer analyst and was active with hobbies including hiking and volleyball. During this period, he suffered from occasional episodes of headache, depression, and complex partial seizures with hallucinatory aura, which were treated intermittently with phenytoin.

Second Presentation and Examination. In October 2000, the 60-year-old patient presented with progressive dizziness, ataxia, and frontal headache. Neurological examination demonstrated decreased attention, lethargic speech, homonymous left upper quadrantanopsia (a known symptom), absence of left pupillary light reaction, papilledema, and ataxic gait. Functionally his KPS score deteriorated to 70 . An emergency $\mathrm{CT}$ scan revealed a right cerebellar tumor that anteriorly displaced the fourth ventricle and caused right-to-left shift of the midline struc-

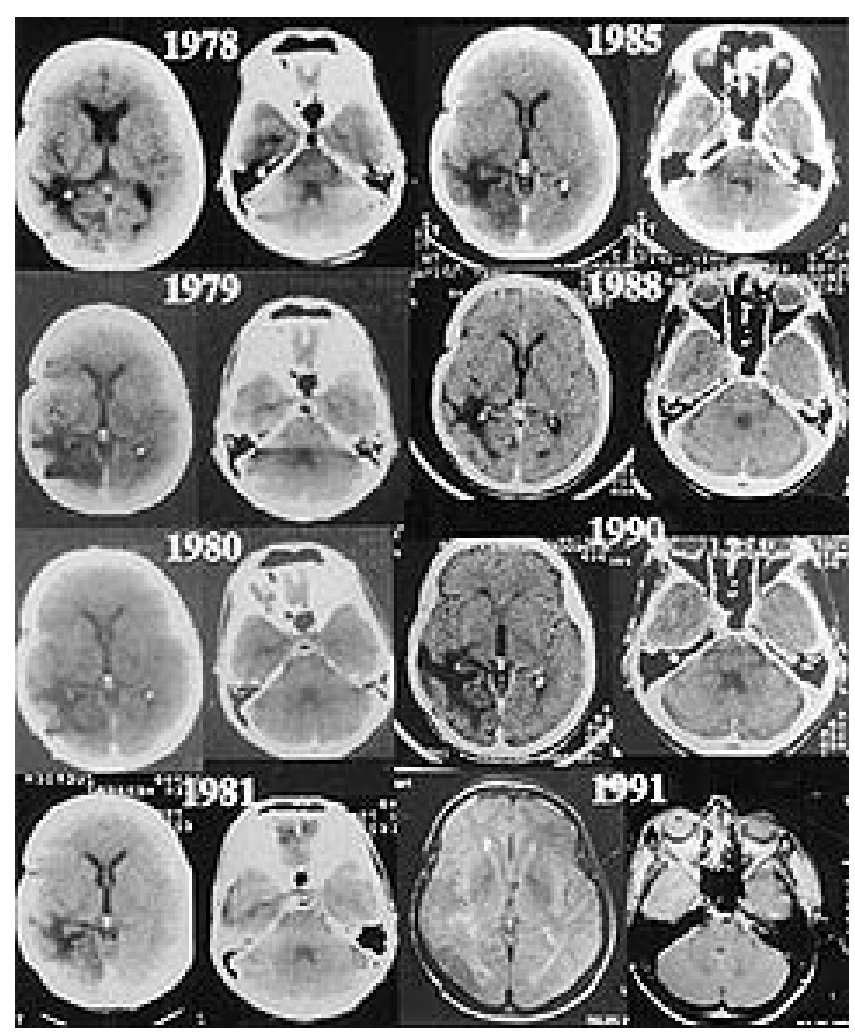

Fig. 1. Follow-up neuroimaging studies obtained between 1978 and 1991. The CT scans (1978-1990) and MR images (1991) reveal no recurrence of the right temporal GBM.

tures and dilation of the third and both lateral ventricles. No calcification was detected. The MR imaging findings confirmed those demonstrated on CT scanning. The tumor was isodense on $\mathrm{T}_{1}$-weighted and hyperintense in $\mathrm{T}_{2}$ weighted images, with moderate heterogeneity (Figs. 2 and 3). Cystic changes were present and the tumor margins were well defined. No recurrence of the right temporal GBM could be demonstrated. As shown in Fig. 3, no evidence of cerebellar disease was visible retrospectively. Preoperative FDG-PET demonstrated high FDG uptake in the right cerebellum and very poor uptake in the right temporal region (Fig. 2). Angiography revealed a hypervascularity in the inferior portion of the tumor. The posterior inferior cerebellar artery was displaced inferiorly. Preoperatively, the patient consented to undergo a right temporal biopsy procedure to obtain tissue from the prior GBM site.

Second Operation. A CT-guided stereotactic biopsy procedure of the GBM resection bed was performed targeting the posterior part of the temporal lobe. Three subcortical specimens could be obtained. The surgeon (J.F.) performed a right paramedian suboccipital craniotomy with the patient in the sitting position. An intraoperatively apparent gross-total tumor resection was achieved with the aid of intraoperative ultrasonography (Fig. 4A). The tumor infiltrated almost the entire right cerebellar hemisphere. The patient could be extubated immediately after surgery, and his cerebellar symptoms and gait improved rapidly. After confirmation of the histological diagnosis, 


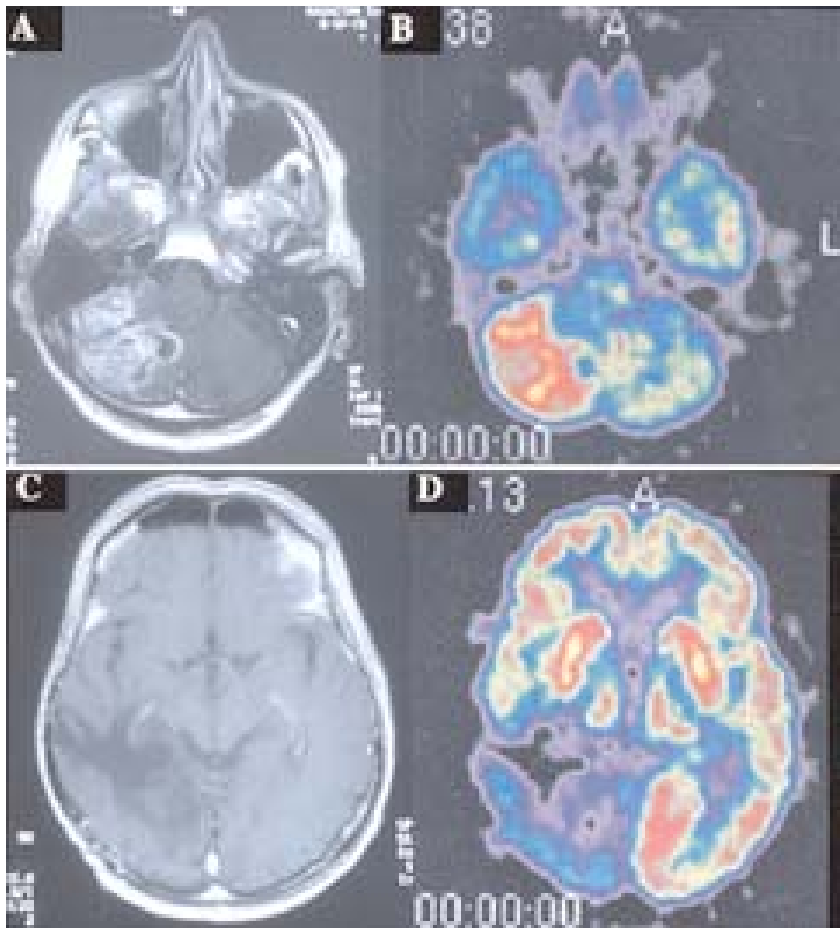

Fig. 2. Axial MR images and FDG-PET scans demonstrating the new tumor located in the right cerebellum (A and B) and no recurrence of the right temporal glioblastoma multiforme (C and D).

MR imaging of the cervical, thoracic, and lumbar spine demonstrated no CSF dissemination of the tumor.

Second Postoperative Course. During the postoperative course the patient developed clinical signs of pulmonary embolism. A lung scintigraphy demonstrated bilateral multisegment and subsegmental pulmonary embolisms, and anticoagulation therapy was performed. The patient was transferred to a neurorehabilitation center; his a KPS score was 80 . The patient refused postoperative radiotherapy and adjuvant chemotherapy. Six months after surgery, MR imaging and FDG-PET scanning demonstrated recurrence of the tumor adjacent to the cerebellopontine angle (Fig. 4B and C). At this point the patient's KPS score was 90. In the following months, the patient deteriorated and developed progressive gait disturbance, dysarthria, and later became somnolent and comatose. He died 9 months after the second surgery.

Pathological Examinations. The surgical specimen obtained 1975 was reexamined. On light microscopic examination, both frozen and paraffin-embedded sections showed a highly cellular tumor, nuclear pleomorphism, multinucleated giant cells, extensive vascular endothelial proliferation, and widespread areas of necrosis (Fig. 5). There was high mitotic activity. Perivascular lymphocytic infiltrates were focally detectable. In addition to this predominating component with characteristic features of GBM, there was a minor component characterized by less cellularity and better differentiation. Molecular genetic analysis was performed retrospectively for this publication. Mutational analysis of exons 5 to 8 of the TP53 tumor suppressor showed no alteration. No EGFR gene amplification was found.

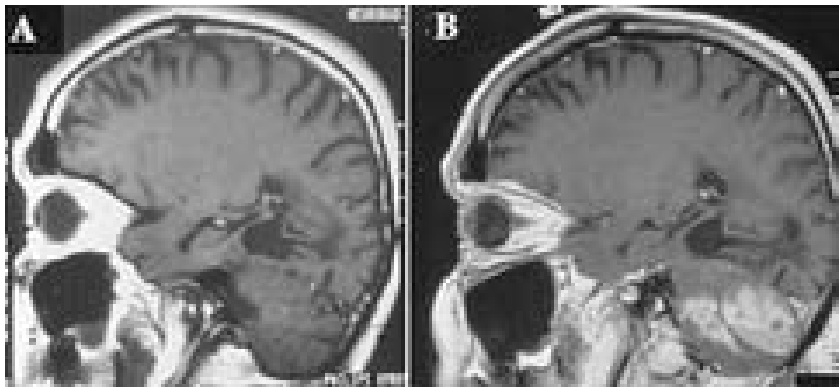

Fig. 3. Sagittal MR images obtained in 1991 (A) and 2000 (B), demonstrating the extent of resection in the right temporal region and no sign of tumor recurrence. The images reveal preservation of the hippocampus and the limits of the resection beneath the inferior horn of right lateral ventricle. No evidence of tumor in the cerebellum was documented in 1991.

The second sampling included two stereotactic biopsy cores obtained in the right temporal region and three fractions of the actual tumor located in the right cerebellar hemisphere. On light microscopic examination of the site of the former GBM (right temporal region), there was insignificant gliosis, but neither GBM nor other glial or nonglial neoplasm was observed (Fig. 6 upper). In contrast, all three biopsy fractions collected from the right cerebellar hemisphere displayed a highly cellular invasive tumor: densely packed small round cells with little cytoplasm and hyperchromatic nuclei (Fig. 6 lower). Apoptotic and mitotic fractions were both high. Rosettes, either of Homer-Wright or Flexner-Wintersteiner type, were inconspicuous, whereas pseudorosettes were prominent. Remnants of the organotypic cerebellar foliage were seen in places, whereas overrun Purkinje cell layers were the only remainder in most parts. Ganglionic differentiation was not a prominent feature; thus, immunoreactivities for chromogranin A, synaptophysin, and neurofilament protein were either absent or very weak for the majority of neoplastic cells, but they were strongly reactive for CD56 and PGP 9.5. At the ultrastructural level, structures resembling synapses were present, whereas dense core granules were a rare finding consistent with negative immunoreactivity for chromogranin A. Hints of glial differentiation were missing. Therefore, a cerebellar recurrence of the former GBM was excluded, and, in the absence of significant reticulin fibers and large cells, the tumor qualified for the classic medulloblastoma Grade IV. Finally, the cytological examination of the cerebrospinal fluid was negative for tumor cells.

\section{DISCUSSION}

We report on a patient who had presented with a right temporal GBM at the age of 35 and underwent resection and fractionated whole-brain radiotherapy. The patient was admitted 26 years later with a medulloblastoma in the right cerebellum (within the radiotherapy field) but no signs of recurrence of the right temporal GBM. Neurodiagnostic studies such as MR imaging, PET scanning, and histopathological examination confirmed the absence of tumor recurrence.

Glioblastoma multiforme is the most malignant astrocytic tumor, composed of poorly differentiated neoplas- 
J. Fandino, et al.

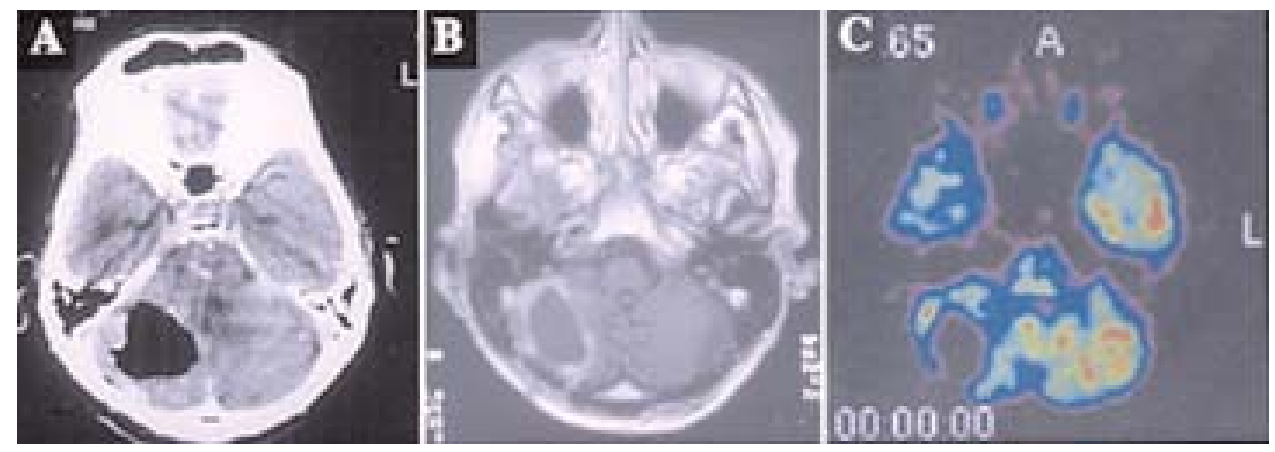

Fig. 4. A: Axial CT scan obtained after resection of the medulloblastoma located in the right cerebellum. B: Axial MR image obtained 6 months later revealing recurrence of the tumor in the anterior limits of the resection hole. C: $\left[{ }^{18} \mathrm{~F}\right]$ fluorodeoxyglucose-PET scan revealing a focal FDG uptake in this region.

tic astrocytes. Histopathological features include cellular polymorphism, nuclear atypia, brisk mitotic activity, vascular thrombosis, microvascular proliferation, and necrosis. ${ }^{3,25}$ Glioblastoma multiforme may develop from WHO Grade II diffuse astrocytomas or anaplastic astrocytomas (secondary glioblastoma), but more frequently they manifest after a short clinical history de novo, without evidence of a less malignant precursor lesion (primary glioblastoma). Glioblastoma multiforme was first described in 1863 by Virchow as a tumor of glial origin, ${ }^{50}$ but the first comprehensive description was provided by Globus and Strauss. ${ }^{17}$ Bailey and Cushing ${ }^{2}$ changed the name from spongioblastoma multiforme to GBM, a term first used by Mallory in $1914 .^{31}$

Survival time in patients with gliomas depends mainly on the phenomenon of malignant progression, which was initially described by Tooth $^{49}$ in 1912 . The prognosis has been a matter of controversy over the past century. In 1926 Bailey and Cushing ${ }^{2}$ wrote: "The impression prevails in both laboratory and clinic that microscopic examination of a specimen removed at operation will not serve to pre-

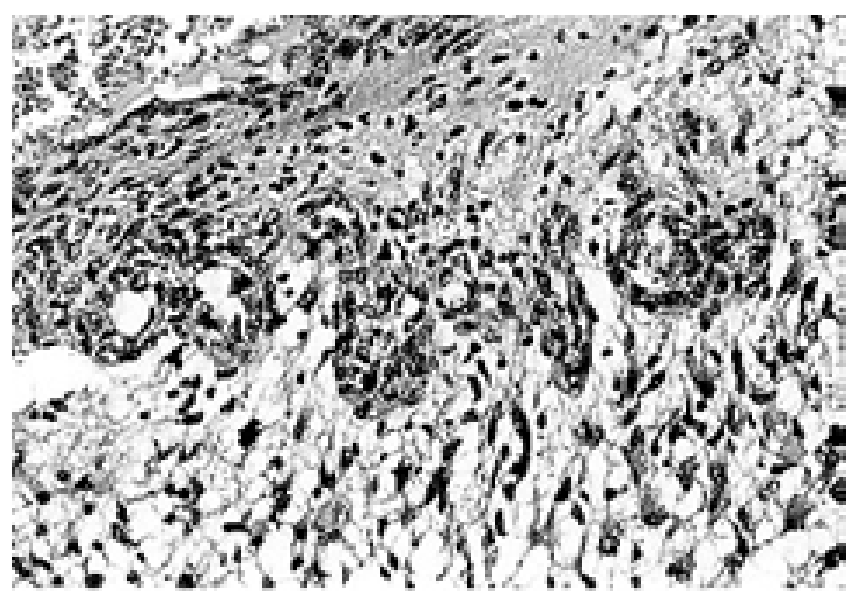

Fig. 5. Photomicrograph showing the results of the specimen obtained in 1975. Both frozen and paraffin-embedded sections showed a malignant glioma with marked cellular and nuclear pleomorphism, multinucleated giant cells, high mitotic activities, extensive vascular endothelial proliferation, and widespread areas of necrosis. $\mathrm{H} \& \mathrm{E}$, original magnification $\times 200$. dict, with a degree of certainty, the future course of development of a true tumor of the brain substance." In contrast, in patients in whom a primary GBM was diagnosed and resected, there is less controversy regarding the survival time. The median duration of survival is approxi-
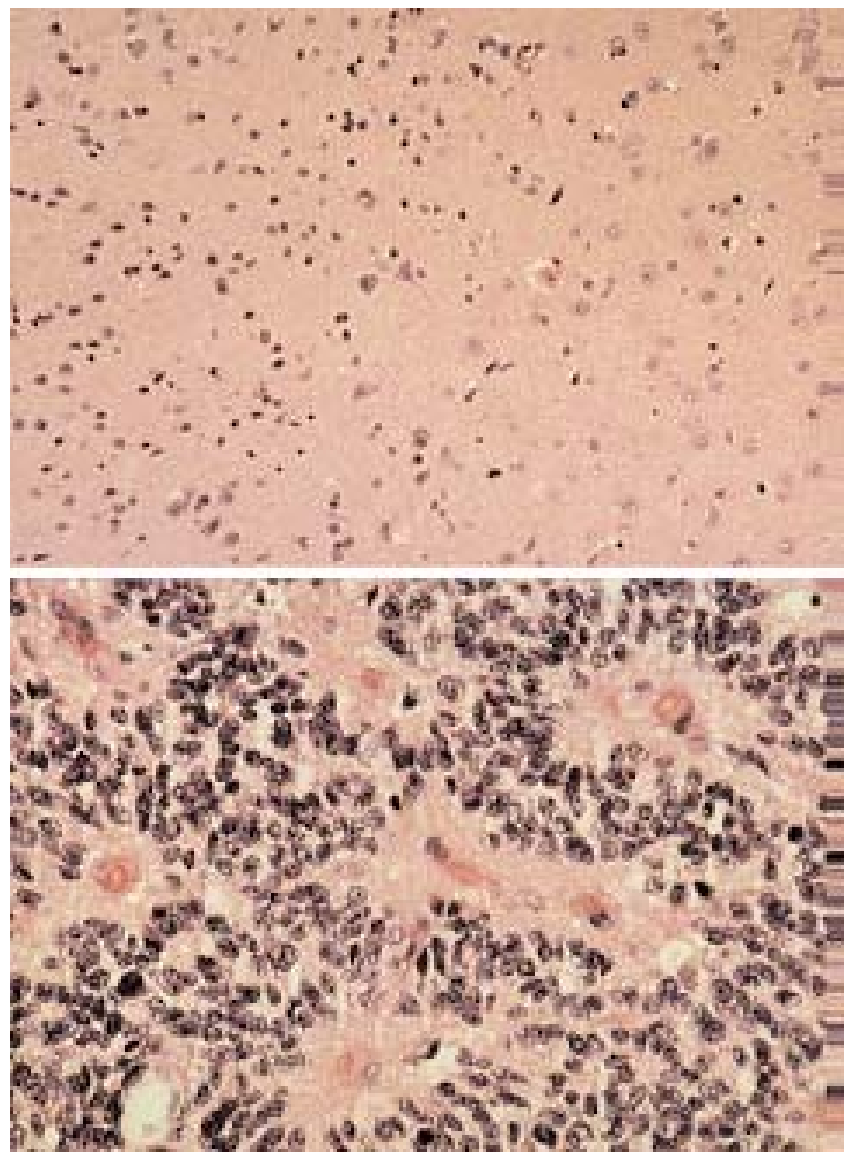

Fig. 6. Photomicrographs. Upper: Biopsy specimen taken from the site of the former GBM (right temporal region) showed neither GBM nor other neoplasm. Lower: A right cerebellar hemispheric biopsy sample with embryonal neoplasm displaying prominent pseudorosettes. $\mathrm{H} \& \mathrm{E}$, original magnifications $\times 50$ (upper) and $\times 100$ (lower). 
mately 50 months, ${ }^{23,35}$ and few patients live more than 5 years. ${ }^{10}$

Long-term survivors of GBM have been anecdotally reported throughout the last 50 years. ${ }^{11,19}$ Recently, a summary of 30 patients who survived 10 years or longer after diagnosis was published (based on cases reported in the literature from 1990-2001). ${ }^{38}$ Despite clinical and genetic studies, factors predictive of clinical outcome in GBM remained an elusive goal. The following six predictive factors have to be considered in long-term survival of GBM: age, KPS score, extent of resection, presence of necrosis, proliferation, and genetic alterations. ${ }^{10,25,41,42}$

In a review of 1003 glioblastoma biopsy specimens studied at the University Hospital Zurich, two thirds (70\%) of the cases involved patients between 45 and 70 years of age (mean age at biopsy procedure 53 years). ${ }^{25}$ More recently, the senior author (M.G.Y.) reported the outcome in a consecutive series of patients with GBM treated at the University Hospital Zurich (1967-1992). ${ }^{52}$ Of 345 patients (male/female ratio 1.6:1) who underwent resection and postoperative radiotherapy, 71 patients $(20.6 \%)$ were younger than 40 years of age. Twenty-two patients $(6.3 \%)$ survived more than 3 years, seven $(2 \%)$ patients survived 5 years, and only two patients $(0.6 \%)$ were alive 10 years after treatment. Interestingly, these two patients were age 31 and 35 years, respectively, at the time of diagnosis. The former case (the only case in which survival was more that 25 years) is presented in this publication. In fact, this finding correlates with previous reports indicating that age is a strong prognostic factor. In patients younger than 45 years of age prognosis is better than in the elderly. ${ }^{7,39,46}$ The reason for this might be an intrinsically more rapid malignant progression in elderly patients ${ }^{43}$ and/or a higher frequency of secondary glioblastomas in younger patients. ${ }^{7,51}$

There is an inverse correlation between KPS score at the time of diagnosis and death rate (number of deaths divided by the total number of patient months of follow up). The ratio of death rate for highest and lowest scores is roughly 3 , which is comparable to the 3.5 ratio demonstrated between older- and younger-age patients. ${ }^{9}$ The duration of preoperative symptoms is also associated with survival. ${ }^{48}$ Chandler, et al., ${ }^{10}$ reviewed data obtained in 449 patients with GBM and studied possible factors associated with long-term survival. Twenty-two patients $(5 \%)$ survived more than 5 years. The authors concluded that long-term survival most likely occurs in those who are young, have high KPS scores, and experience a long disease-free interval after the initial diagnosis. In 20 of 22 patients the KPS score was 80 or greater. Our patient was initially treated at age 35 years but presented with a low KPS score prior to the surgery. Nevertheless, his KPS score improved to 90 postoperatively and remained unchanged during the next 25 years.

The extent of resection as a predictive factor in the prognosis of patients with GBM remains controversial. Despite the fact that a significant survival difference has not been reported in all studies of cases in which the extent of resection has been great, ${ }^{29}$ there is some evidence that complete resection favors longer survival. ${ }^{1,2,4-7}$ The authors of one study demonstrated that in cases in which postoperative MR imaging demonstrated residual tumor, there was a significantly higher (6.6-fold) risk of death (shorter survival time) than in those in which the entire contrast-enhancing tumor was shown to been removed. ${ }^{1}$

Although the first CT study in our case could be performed almost 3 years after surgery in 1978, an enhancing lesion was not visualized in the right temporal region at that time nor on the subsequent $\mathrm{CT}$ and MR imaging studies performed until 2000 (Figs. 1-3). Clearly in our case, surgical cytoreduction followed by radiotherapy led to a complete imaging-documented response, as evidenced by subsequent CT and MR imaging findings. Even if longterm survival after GBM resection is possible, the potential benefits of maximal resection must be balanced against the potential function-related effects on postoperative quality of life. New technological advances such as fluorescence-guided resection and intraoperative real-time MR imaging are promising advances to assist in safely achieving maximal tumor resection. ${ }^{14,47}$

The correct histopathological recognition of GBM has prognostic and therapeutic implications, because oligodendroglial tumors are more likely to respond favorably to chemotherapy. Histopathological reevaluation of patients harboring GBM in whom survival is long term should be considered mandatory. Kraus, et al., ${ }^{28}$ reviewed a total of 52 cases of GBM in which survival was 12 months or greater and reported that 13 tumors $(25 \%)$ were reclassified as anaplastic oligodendroglioma (WHO Grade III; seven cases) or anaplastic oligoastrocytoma (WHO Grade III; two cases). In addition, three were reclassified as anaplastic astrocytoma, and one was identified as anaplastic pilocytic astrocytoma. ${ }^{28}$ The histopathological reevaluation of the tumor in our case confirmed the diagnosis of GBM.

The presence and extent of necrosis in GBM is associated with poor clinical outcome. ${ }^{3,7,34}$ In our patient's tumor the area of necrosis was smaller than that usually found in elderly patients, reflecting a possible factor influencing the long-term survival in this case. With respect to proliferation, the tumor originally obtained in 1975 showed marked cellular and nuclear pleomorphism, multinucleated giant cells, high mitotic activity, and extensive vascular endothelial proliferation. Although these findings might correlate retrospectively with the clinical grade, they do not correlate with the outcome in our case. ${ }^{8,24,33,36}$ The presence of multinucleated giant cells may have contributed to a better outcome compared with that in other GBM cases. ${ }^{7,38}$

In our case molecular genetic analysis revealed no altered expression of TP53 and EGRF. Although some investigators have reported favorable prognosis in GBM if molecular alterations such as an increased frequency of TP53 mutations and deletions of chromosomal arm 17p are present, ${ }^{32,41,51}$ and poorer prognosis if amplification with overexpression of EGFR is present, ${ }^{12,21}$ others have not confirmed these findings. , $26,27,40$ Recently, Simmons, et al., ${ }^{44}$ analyzed tumor tissue obtained in 110 cases of newly diagnosed GBM from two clinical protocols. Similar to results in other studies, the authors found no association of EGFR overexpression (as assessed by immunohistochemistry), p53 immunopositivity, or p53 mutation with survival in the entire sample. Epidermal growth factor receptor overexpression, however, showed trends toward a worse prognosis in patients younger than the median age but a better prognosis in those older than 
the median age. In younger patients, they identified a statistically significant association of EGFR overexpression with worse survival in the p53-negative but not p53-positive tumors. In addition, the authors found that EGFR overexpression was negatively associated with survival only in the p53 wild-type cases. This complex relationship between EGFR and p53 in younger patients was also present in a multivariate analysis that incorporated additional prognostic variables. These results suggest that analysis of prognostic markers in GBM is complex, and maximal information may require analysis of subgroups based on age and the status of specific markers such as p53.

The patient described in this report presented at the age of 60 years with a cerebellar tumor 26 years after resection of a right temporal GBM. The MR image 9 years prior to this diagnosis indicated no lesion in the cerebellum (Fig. 3). Based on MR imaging, FDG-PET scanning, and histopathological findings, the tumor was interpreted as a de novo medulloblastoma. Medulloblastomas are malignant, invasive embryonal tumors of the cerebellum with preferential manifestation in children, predominant neuronal differentiation, and an inherent tendency to metastasize via CSF pathways. ${ }^{15}$ Seventy percent of medulloblastomas occur in individuals younger than 16 years of age. ${ }^{4,15}$ In adulthood, $80 \%$ of medulloblastomas arise in the 21 to 40 -year-old age group. ${ }^{16,22}$ The estimated annual incidence of medulloblastoma in adults is 0.05 per $100 .{ }^{4} \mathrm{Be}-$ cause this tumor rarely occurs beyond the fifth decade, our case is relevant for the neurosurgery literature. Of 89 consecutive cases with medulloblastoma treated at the University Hospital Zurich, only two patients $(2.3 \%)$ were 60 years of age or older. ${ }^{15}$ Cerebellar medulloblastoma diagnosed in our 60-year-old patient is undoubtedly a very uncommon finding in neurosurgical practice. It is difficult to address the role of radiotherapy in the pathogenesis of the medulloblastoma after such a long-term followup period in this case. ${ }^{6}$ Because postradiation vascular changes in the white matter were not observed in the specimen, it can be assumed that the medulloblastoma was a de novo tumor.

Few data are available regarding treatment of adult medulloblastoma because its incidence is low, and most published series have concerned small samples and/or have been collected over a long period of time. ${ }^{4}$ It is widely debated whether medulloblastoma in adults has a different prognosis from that in children. In some series the prognosis in adults was reported as the same as that in children, ${ }^{2,4}$ whereas others have reported better survival in adults because recurrences appear earlier in children.,20,37 Long-term survival is possible in adults with medulloblastoma; the reported rates range from 50 to $84 \%$ at 5 years and 40 to $50 \%$ at 10 years. ${ }^{2,3,6,8}$ Because the data in the literature are based on retrospective studies, treatments are neither randomized nor uniform; however, some treatment cornerstones have been identified. The first step is surgery, in which the resection should be as radical as possible; adjuvant 55-Gy radiotherapy must be targeted at the posterior fossa, with 36 Gy to the remaining cranial-spinal axis; adjuvant chemotherapy may be useful in patients at high risk of recurrence provided that it is administered before radiotherapy in moderate-high dosages and includes cisplatin, etoposide, and cyclophosphamide. ${ }^{6} \mathrm{Al}-$ though radiotherapy targeting the posterior fossa is stan- dard, craniospinal radiotherapy is now controversial and many therapists restrict its use to high-risk patients. Chemotherapy is standard and can be administered before or after radiotherapy. ${ }^{6}$

Recently, 57 adult patients with medulloblastoma were evaluated at the University of Texas M.D. Anderson Cancer Center over a 20-year period (1978-1998). ${ }^{30}$ Statistical analysis of prognostic factors and overall survival was performed for a subgroup of 28 patients who were followed exclusively at their institution, between the time of diagnosis and death or last follow-up visit. Overall survival in these 28 patients was $91 \%$ at 3 years and $84 \%$ at 5 years, whereas median survival was not reached after a median follow-up period of 168 weeks (range 9-602 weeks). The authors reported a progression-free survival for all patients of $68 \%$ at 3 years and $62 \%$ at 5 years. Univariate analysis of clinical features, such as age, sex, extent of local disease, extent of resection, and use of adjuvant chemotherapy, did not identify any prognostic variables for survival among the 28 patients. In this retrospective study, adult medulloblastoma appeared to be associated with a favorable prognosis after treatment with maximally feasible resection followed by craniospinal irradiation. Considering the marked difference between survival and progression-free survival, the authors concluded that salvage therapy, usually involving combination chemotherapy, is of benefit. Nevertheless, results of adjuvant chemotherapy are inconclusive in most of the studies. ${ }^{6}$ Greenberg, et al. ${ }^{18}$ recently reviewed the cases of 17 adult patients with medulloblastoma who underwent surgery, craniospinal radiotherapy plus local boost, and one of two adjuvant chemotherapy regimens. Ten patients underwent treatment involving the "Packer protocol," which consisted of craniospinal radiotherapy plus weekly vincristine followed by eight cycles of cisplatin, lomustine, and vincristine. In seven patients POG protocol was applied, consisting of alternating courses of cisplatin/etoposide and cyclophosphamide/vincristine, followed by craniospinal radiotherapy. Median relapse-free survival for patients on the Packer protocol was 26 months, whereas for those on the POG regimen it was 48 months. The authors reported that POG and Packer protocols did not have a statistically significant difference in relapse-free or overall survival because of the small sample size. Because our patient refused further postoperative treatment, the possible benefits of craniospinal radiotherapy and adjuvant chemotherapy could not be evaluated. The patient experienced a 24 -week progression-free period and survived 37 weeks after removal of the tumor.

\section{Acknowledgments}

The authors thank Professor Paul Kleihues and Dr. Hirokko Ohgaki from the International Agency for Research on Cancer, Lyon, France, for the reevaluation of the histological examination performed in 1975, and genetic molecular analysis, respectively. We are indebted to Professor M. Linskey for his suggestions during the preparation of this manuscript.

\section{References}

1. Albert FK, Forsting M, Sartor K, et al: Early postoperative magnetic resonance imaging after resection of glioma: objective evaluation of residual tumor and its influence on regrowth and prognosis. Neurosurgery 34:45-61, 1994 
2. Bailey P, Cushing H: A Classification of the Tumors of the Glioma Group on a Histogenetic Basis with a Correlated Study of Prognosis. Philadelphia: JB Lippincott, 1926

3. Barker FG II, Davis RL, Chang SM, et al: Necrosis as a prognostic factor in glioblastoma multiforme. Cancer 77: 1161-1166, 1996

4. Bloom HJ, Bessell EM: Medulloblastoma in adults: a review of 47 patients treated between 1952 and 1981. Int J Radiat Oncol Biol Phys 18:763-772, 1990

5. Bouvier-Labit $\mathrm{C}$, Chinot O, Ochi $\mathrm{C}$, et al: Prognostic significance of Ki67, p53 and epidermal growth factor receptor immunostaining in human glioblastomas. Neuropathol Appl Neurobiol 24:381-388, 1998

6. Brandes AA, Palmisano V, Monfardini S: Medulloblastoma in adults: clinical characteristics and treatment. Cancer Treat Rev 25:3-12, 1999

7. Burger PC, Green SB: Patient age, histologic features, and length of survival in patients with gliblastoma multiforme. Cancer 59:1617-1625, 1987

8. Burger PC, Shibata T, Kleihues P: The use of monoclonal antibody Ki-67 in the identification of proliferating cells: aplication to surgical neuropathology. Am J Surg Pathol 10:611-617, 1986

9. Byar DP, Green SB, Strike TA: Prognostic factors for malignant glioma, in Walker MD (ed): Oncology of the Nervous System. Boston: Martinus Nijhof Publishers, 1983, pp 379-395

10. Chandler KL, Prados MD, Malec M, et al: Long-term survival in patients with glioblastoma multiforme. Neurosurgery 32: 716-720, 1993

11. de Tribolet N, Deruaz JP, Zander E: Familial gliomas. Neurochirurgia (Stuttg) 22:225-228, 1979

12. Eppenberger U, Muller H: Growth factor receptors and their ligands. J Neurooncol 22:249-254, 1994

13. Forsyth PA, Cairncross JG: Treatment of malignant glioma in adults. Curr Opin Neurol 8:414-418, 1995

14. Gering DT, Nabavi A, Kikinis R, et al: An integrated visualization system for surgical planning and guidance using image fusion and an open MR. J Magn Reson Imaging 13:967-975, 2001

15. Giangaspero F, Bigner SH, Kleihues P, et al: Medulloblastoma, in Kleihues P, Cavenee WK (eds): Pathology and Genetics of Tumors of the Nervous System. Lyon: IARC Press, 2000, pp 129-137

16. Giordana MT, Schiffer P, Lanotte M: Epidemiology of adult medulloblastoma. Int J Cancer 80:689-692, 1999

17. Globus JH, Strauss I: Spongioblastoma multiforme. A primary malignant form of brain neoplasm: its clinical and anatomic features. Arch Neurol Psychiatry 14:139-151, 1925

18. Greenberg HS, Chamberlain MC, Glantz MJ, et al: Adult medulloblastoma: multiagent chemotherapy. Neuro-oncol 3: 29-34, 2001

19. Gutin PH, Leibel SA, Wara WM, et al: Recurrent malignant gliomas: survival following interstitial brachytherapy with highactivity iodine-125 sources. J Neurosurg 67:864-873, 1987

20. Hartsell WF, Montag AG, Lydon J, et al: Treatment of medulloblastoma in adults. Am J Clin Oncol 15:207-211, 1992

21. Hiesiger EM, Hayes RL, Pierz DM, et al: Prognostic relevance of epidermal growth factor receptor (EGF-R) and c-neu/erbB2 expression in glioblastomas (GBMs). J Neurooncol 16: 93-104, 1993

22. Hubbard JL, Scheithauer BW, Kispert DB, et al: Adult cerebellar medulloblastomas: the pathological, radiographic, and clinical disease spectrum. J Neurosurg 70:536-544, 1989

23. Imperato JP, Paleologos NA, Vick NA: Effects of treatment on long-term survivors with malignant astrocytomas. Ann Neurol 28:818-822, 1990

24. Karamitopoulou E, Perentes E, Diamantis I, et al: Ki-67 immonoreactivity in human central nervous system tumors: a study with MIB 1 monoclonal antibody on archival material. Acta Neuropathol 87:47-54, 1994
25. Kleihues P, Burger PC, Collins VP, et al: Glioblastoma, in Kleihues P, Cavenee WK (eds): Pathology and genetics of tumors of the nervous system. Lyon: IARC Press, 2000, pp $29-40$

26. Kraus JA, Glesmann N, Beck M, et al: Molecular analysis of the PTEN, TP53 and CDKN2A tumor suppressor genes in longterm survivors of glioblastoma multiforme. J Neurooncol 48: 89-94, 2000

27. Kraus JA, Wenghoefer M, Glesmann N, et al: TP53 gene mutations, nuclear $\mathrm{p} 53$ accumulation, expression of Waf/p21, Bcl-2, and CD95 (APO-1/Fas) proteins are not prognostic factors in de novo glioblastoma multiforme. J Neurooncol 52:263-272, 2001

28. Kraus JA, Wenghoefer M, Schmidt MC, et al: Long-term survival of glioblastoma multiforme: importance of histopathological reevaluation. J Neurol 247:455-460, 2000

29. Kreth FW, Warnke PC, Scheremet R, et al: Surgical resection and radiation therapy versus biopsy and radiation therapy in the treatment of glioblastoma multiforme. J Neurosurg 78: 762-766, 1993

30. Kunschner LJ, Kuttesch J, Hess K, et al: Survival and recurrence factors in adult medulloblastoma: the M.D. Anderson Cancer Center experience from 1978 to 1998. Neuro-oncol 3: 167-173, 2001

31. Mallory FB: Principles of pathologic histology. Philadelphia: WB Saunders, 1925

32. Meyer-Puttlitz B, Hayashi Y, Waha A, et al: Molecular genetic analysis of giant cell glioblastoma. Am J Pathol 151:853-857, 1997

33. Montine TJ, Vandersteenhoven JJ, Aguzzi A, et al: Prognostic significance of Ki-67 proliferation index in supratentorial fibrillary asrtocytic neoplasms. Neurosurgery 34:674-678, 1994

34. Nelson JS, Tsukada Y, Schoenfeld D, et al: Necrosis as a prognostic criterion in malignant supratentorial, astrocytic gliomas. Cancer 52:550-554, 1983

35. Netsky MG, August B, Fowler W: The longevity of patients with glioblastoma multiforme. J Neurosurg 7:261-269, 1950

36. Raghavan R, Steart PV, Weller RO: Cell proliferation patterns in the diagnosis of astrocytomas, anaplastic astrocytomas and glioblastoma multiforme: a Ki-67 study. Neuropathol Appl Neurobiol 16:123-133, 1990

37. Rochkind S, Blatt I, Sadeh M, et al: Extracranial metastases of medulloblastoma in adults: literature review. J Neurol Neurosurg Psychiatry 54:80-86, 1991

38. Sabel M, Reifenberger J, Weber RG, et al: Long-term survival of a patient with giant cell glioblastoma. Case report. J Neurosurg 94:605-611, 2001

39. Salmon I, Dewitte O, Pasteels JL, et al: Prognostic scoring in adult astrocytic tumors using patient age, histopathological grade, and DNA histogram type. J Neurosurg 80:877-883, 1994

40. Sano $\mathrm{T}$, Lin $\mathrm{H}$, Chen $\mathrm{X}$, et al: Differential expression of MMAC/PTEN in glioblastoma multiforme: relationship to localization and prognosis. Cancer Res 59:1820-1824, 1999

41. Schmidt MC, Antweiler S, Urban N, et al: Impact of genotype and morphology on the prognosis of glioblastoma. J Neuropathol Exp Neurol 61:321-328, 2002

42. Scott JN, Rewcastle NB, Brasher PM, et al: Which glioblastoma multiforme patient will become a long-term survivor? A population-based study. Ann Neurol 46:183-188, 1999

43. Shafqat S, Hedley-Whyte ET, Henson JW: Age-dependent rate of anaplastic transformation in low-grade astrocytoma. Neurology 52:867-869, 1999

44. Simmons ML, Lamborn KR, Takahashi M, et al: Analysis of complex relationships between age, p53, epidermal growth factor receptor, and survival in glioblastoma patients. Cancer Res 61:1122-1128, 2001

45. Simpson JR, Horton J, Scott C, et al: Influence of location and extent of surgical resection on survival of patients with glioblastoma multiforme: results of three consecutive Radiation 
Therapy Oncology Group (RTCOG) clinical trials. Int J Radiat Oncol Biol Phys 26:239-244, 1993

46. Sneed PK, Prados MD, McDermott MW, et al: Large effect of age on the survival of patients with glioblastoma treated with radiotherapy and brachytherapy boost. Neurosurgery 36: 898-904, 1995

47. Stummer W, Novotny A, Stepp H, et al: Fluorescence-guided resection of glioblastoma multiforme by using 5-aminolevulinic acid-induced porphyrins: a prospective study in 52 consecutive patients. J Neurosurg 93:1003-1013, 2000

48. Takeuchi K, Hoshino K: Statistical analysis of factors affecting survival after glioblastoma multiforme. Acta Neurochir 37: 57-73, 1977

49. Tooth HH: Some observations on the growth and survival-period of intracranial tumours, based on the records of 500 cases, with special reference to the pathology of the gliomata. Original articles and clinical cases. Brain Pathol 35 (Issue 2):61-108, 1912
50. Virchow R: Die Kranklhaften Geschwülste. Berlin: Verlag von August Hirschwald, 1863

51. Watanabe K, Tachibana O, Sato K, et al: Overexpression of the EGF receptor and p53 mutations are mutually exclusive in the evolution of primary and secondary glioblastomas. Brain Pathol 6:217-223, 1996

52. Yaşargil MG: Patient population and outcome from individual glioma groups, in Yaşargil M: Microneurosurgery of CNS Tumors Stuttgart: Thieme M, 1996, Vol IVB, pp 344-361

Manuscript received November 21, 2002.

Accepted in final form February 20, 2003.

Address reprint requests to: Javier Fandino, M.D., Department of Neurosurgery, University Hospital Bern, 3010 Bern, Switzerland. email: fandino@bluewin.ch. 